\title{
Woman Safety Band using IOT
}

\author{
Samal A ${ }^{1}$, K. Akshai Kanth ${ }^{2}$, A. Navaneethan ${ }^{3}$, J. Suhash ${ }^{4}$ \\ ${ }^{1}$ UG - Computer Science and Engineering, Akshaya College of Engineering and Technology, Coimbatore, Tamil Nadu \\ ${ }^{2}$ UG - Computer Science and Engineering, Akshaya College of Engineering and Technology, Coimbatore, Tamil Nadu \\ ${ }^{3}$ UG - Computer Science and Engineering, Akshaya College of Engineering and Technology, Coimbatore, Tamil Nadu \\ ${ }^{4}$ UG - Computer Science and Engineering, Akshaya College of Engineering and Technology, Coimbatore, Tamil Nadu
}

\begin{abstract}
The foremost purpose of this paper is to introduce the concept of a women's guard for application in India. The foremost purpose of this device is to act as an emergency device for girls who are in potential danger of being attacked. The woman possessing this device will press the push if in peril. An SMS containing the latitude and longitude coordinates are getting to be sent to preferred mobile numbers informing them of the danger and thus the situation. The received coordinates are often viewed on google maps to figure out things of the woman and appropriate help are often provided. This concept was devised within the wake of great crime against women in India and to help curb those crimes. Our system also detects the guts beat of the person and blood heat. It'll used when the surveillance person was unable to trigger the Button.
\end{abstract}

Keywords: Safety, women, push, Heartbeat, Body temperature, GSM

\section{INTRODUCTION}

Women's safety in India has become a concerning issue since 2012 with crimes against women growing at an alarming rate. [1]Crimes like kidnapping, harassment towards women and young girls have reached unfathomable levels. The National Crime Records Bureau (NCRB), 2015 has reported that the conviction rate of crimes against women in India may be a smaller amount than 22\%. The cases of crime against women have totalled to 327,394, as reported in 2015. this statistics show a 3.1\% decline from 2014. the absolute best conviction rate that has ever been reported was under the Immoral Traffic Prevention Act (49\%). it had been followed by the Protection of women from violence Act (47.8\%). Abetment to suicide and Cruelty by Husband and his Relatives was reported to possess the lowest conviction rate.

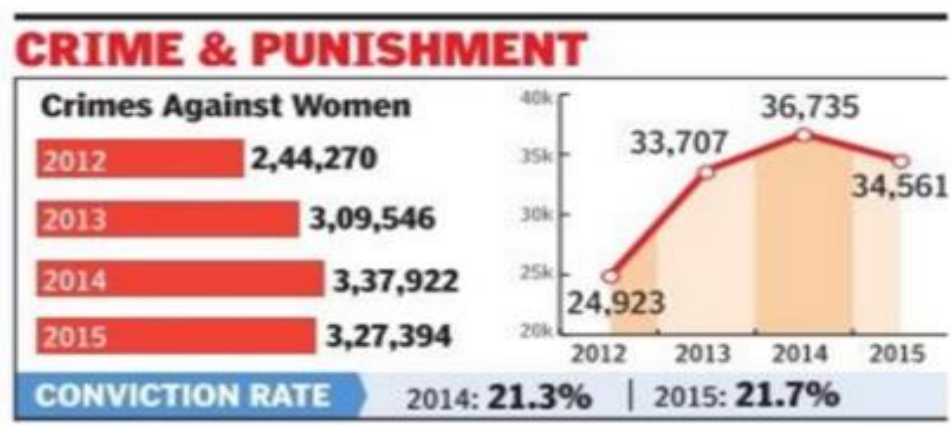

Fig 1. [2] Crime against women statistics

According to the above figure, crime against women [2] has increased once a year. From 2012 to 2013 , the cases increased from 2, 44,270 to 3.09,546. The cases increased from 2013 to 2014 then decreased slightly in 2015 . The conviction rate was increased only by $0.7 \%$ from 2014 to 2015 . The device proposed during this paper is based on a tool developed to form sure child safety [3]. the child guard could also be a wearable device through which folks can keep track of their children.

\section{EXPERIMENTAL METHODS OR METHODOLOGY}

The proposed device will work as an emergency device for girls in distress. the hardware components of this device is an Arduino Uno microcontroller and a GSM module, Pulse sensor,LM35. this technique also detects the blood heat and 


\section{International Advanced Research Journal in Science, Engineering and Technology}

Vol. 8, Issue 6, June 2021

\section{DOI: $10.17148 / I A R J S E T .2021 .8638$}

heart beat of the source person. this wearable device was connected to our main system through the RF transmitter and Receiver.

[4]GSM stands for Global System for mobile Communications. The technology behind the worldwide System for Mobile communication $\left(\mathrm{GSM}^{\mathrm{TM}}\right)$ uses modulation of Phase Shift Keying (PSK) which can be a variant of Gaussian Minimum Shift Keying (GMSK) with Time Division Multiple Access (TDMA) signalling over Frequency Division Duplex (FDD) carriers. GSM was designed principally for voice telephony, but a spread of bearer services were defined like ISDN, allowing circuit switched data connections up to 9600 data bits/s. General Packet Radio Service (GPRS) was developed a few of years later allowing packet switched applications thus enabling access to internet and conjunction of multiple carriers for higher speed. The GPRS offerings were commercially introduced first within the first 2000's.

[5] Arduino could also be a platform on which integration of hardware and software is implemented. It offers a series of analog and digital inputs. the event environment is freely available for all platforms and is certainly programmable. The environment is additionally highly flexible. The GSM module used for the device could also be a sim808 module. it's highly compatible with ATmega 328p microcontroller. The device is activated by the woman in distress by pressing a push. The push activates the GSM module for communication. The module consists of a daily SIM card which may be used for calling and SMS purposes. The module will send the coordinates of the woman to the stored mobile numbers within the device. The concept behind the device is additionally to introduce a price efficient device. The device should be affordable by the standard Indian consumer. As far India cares, a more complex solution to the matter wouldn't be advisable within the present time, because it increases the worth of the device tremendously. the standard Indian consumer won't be able to afford or invest in it. it is a known undeniable fact that majority of victims are below or on the poverty line. Therefore implementing the discussed device are often economical for the standard Indian consumer. Therefore to develop a more efficient device, the worth of manufacturing should be normalized.

\subsection{HARDWARE ARCHITECTURE}

The subsequent are the hardware components of the device:

A.

Arduino Uno

The microcontroller used is an ATmega 328p microcontroller. it's one chip microcontroller of the megaAVR family. The [6] microcontroller are often divided into two parts- hardware and software. The hardware consists of the next features- the USB plug which is used to upload the program into the board. It can also be used as a source of power supply. The plug supplies a regulated voltage of $5 \mathrm{~V}$. just just in case it's insufficient, an external power supply of 9 to $12 \mathrm{~V}$ are often given. There are 5 analog pins (A0 to A5). The digital i/o pins are from 2 to 13 . The push is used to reset the microcontroller so on run a fresh program. There are $3.3 \mathrm{~V}$ and $5 \mathrm{~V}$ power pins to power the Arduino. The second part is that the software. The software involves sending commands and directions to talk with and run the hardware.

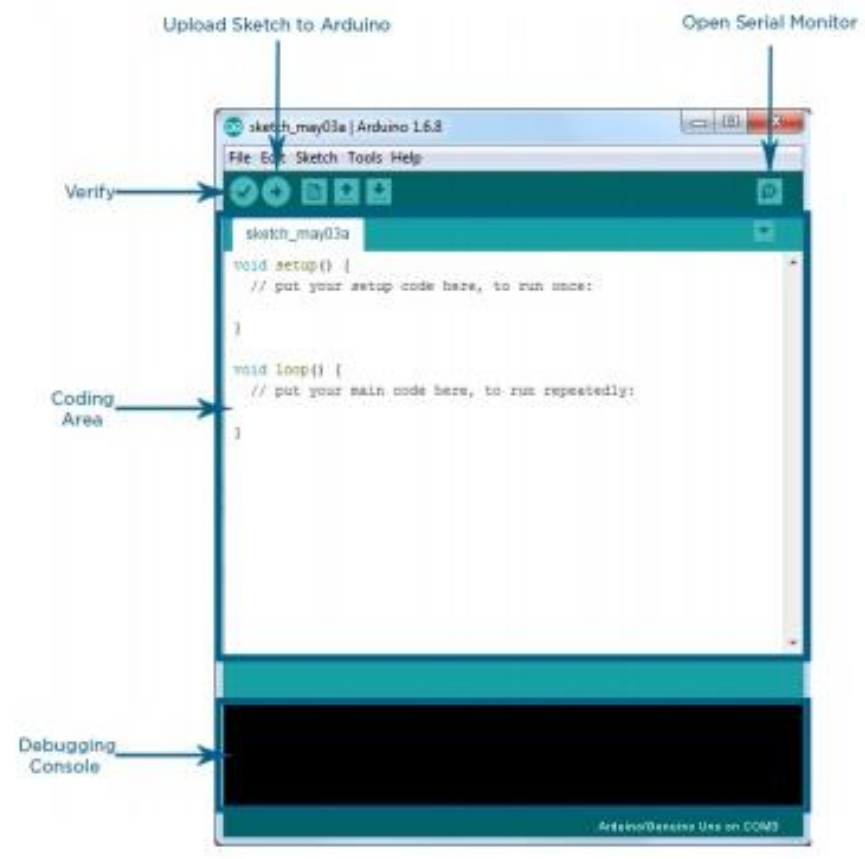

Fig 2.[7] IDE window 


\section{International Advanced Research Journal in Science, Engineering and Technology}

Vol. 8, Issue 6, June 2021

\section{DOI: $10.17148 / I A R J S E T .2021 .8638$}

The software environment used to run Arduino is that the Arduino IDE(Integrated Development Environment). The components under IDE are- message window area, command area and text area. The command area constitutes the menu of tools like File, Edit, Sketch, Tools and Help. The programs are often saved by clicking on the Save button. The text area is that the space to write down down the code. Embedded $\mathrm{C}$ language is that the most typical language used to develop the code or program. By default there is a setup function which is used to initialize variables. there's also a loop subroutine present to feature the foremost code in it. The message window area is used to means the state of the execution of the program.

\section{B. GSM module}

The GSM module used could also be a [8] sim808 module. it is a GSM/GPRS module with quad band which performs satellite communication using GPS technology. Both GPRS and GPS are integrated into a compact SMT package thus saving both time and price for patrons using the module to develop applications. It features an industrystandard interface which allows the tracking of variable resources at any location seamlessly. the general features of the GSM module includes a quad band of $850 / 900 / 1800 / 1900 \mathrm{MHz}$ and a GPRS multi-slot class $12 / 10$. It belongs to GPRS mobile station class B which is Compliant to GSM phase 2/2+ and class 4 (2 W @ 850/900MHz) and class 1 (1 W @ $1800 / 1900 \mathrm{MHz}$. it is also Bluetooth compliant with 3.0+EDR. The FM range is $76-109 \mathrm{MHz}$ worldwide bands which have a $50 \mathrm{KHz}$ tuning step. the dimensions of the module are $24 \times 24 \times 2.6 \mathrm{~mm}$, weighing $3.30 \mathrm{~g}$. it's controlled by AT commands which are 3GPP TS 27.007, 27.005 and SIMCOM enhanced AT Commands. the supply voltage ranges from $3.4-4.4 \mathrm{~V}$. the power consumption by the module is low. The operating temperature ranges from $40^{\circ} \mathrm{C}-85^{\circ} \mathrm{C}$. For SMS via GSM/GPRS, the specifications are point to point MO and MT, SMS cell broadcast and Text and PDU mode. The specification for GPRS data could also be a GPRS class 12, that the utmost downlink/uplink speed is $85.6 \mathrm{kbps}$. It also has PBCCH support. The coding schemes are CS 1, 2, 3, 4, PPP-stack. The CSD can go up to $14.4 \mathrm{kbps}$. There are connection ports for a microphone and a speaker, in conjunction with four other ports. These ports are TX, RX, ground and $5 \mathrm{~V}$ power supply. The TX and RX ports are the ports which may be connected to the Arduino microcontroller. Both will have footing.

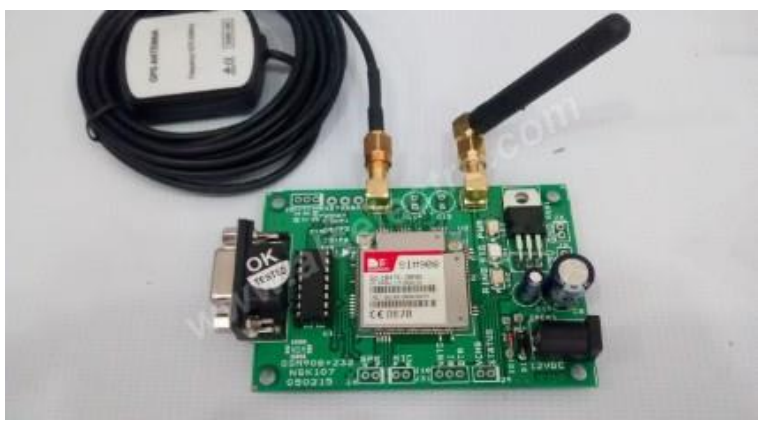

C. Pulse sensor

Pulse Sensor may be a well-designed plug-and-play heart-rate sensor for Arduino. It are often employed by students, artists, athletes, makers, and game \& mobile developers who want to simply incorporate live heart- rate data into their projects. ... It also includes an open-source monitoring app that graphs your pulse in real time.

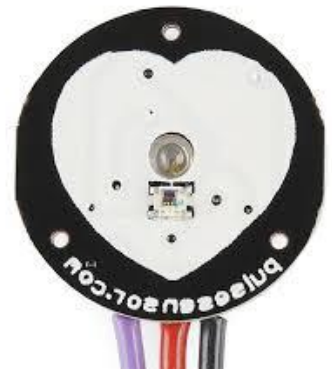

Fig3. Pulse sensor

\section{LM35}

The temperature sensor commonly mentioned as LM35 is used to sense this temperature of the environment. The LM35 series are precision integrated-circuit temperature devices with an output voltage linearly-proportional to the Centigrade temperature. The LM35 device features a plus over linear temperature sensors calibrated in Kelvin, because the user isn't required to subtract an outsized constant voltage from the output to urge convenient Centigrade scaling. 


\section{International Advanced Research Journal in Science, Engineering and Technology}

Vol. 8, Issue 6, June 2021

\section{DOI: $10.17148 / I A R J S E T .2021 .8638$}

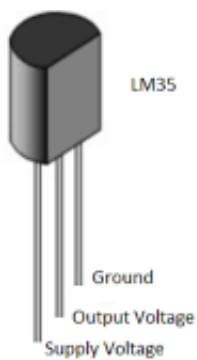

Fig4. LM35

An RF module (short for radio-frequency module) may be a (usually) small device want to transmit and/or receive radio signals between two devices. In an embedded system it's often desirable to speak with another device wirelessly. This wireless communication could also be accomplished through optical communication or through radiofrequency $(\mathrm{RF})$ communication. for several applications, the medium of choice is RF since it doesn't require line of sight. RF communications incorporate a transmitter and a receiver. they're of varied types and ranges. Some can transmit up to 500 feet.

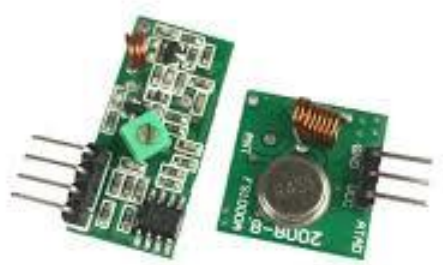

Fig 5. RF Transmitter And receiver

\section{F. $\quad$ Push Button}

Push buttons allow us to power the circuit or make any particular connection only we press the button. Simply, it makes the circuit connected when pressed and breaks when released. A push is additionally used for triggering of the SCR by gate terminal

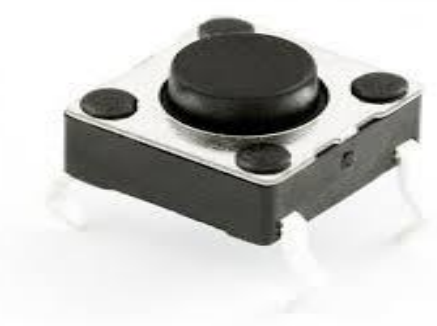

Fig6. Push (or) Emergency Button

\subsection{SYSTEM DESIGN}

GSM Arduino Interfacing

The Arduino Uno is interfaced with the GSM module using two pins, pins 9 and 10, connected to the tx and rx pins of the module respectively. The module is powered up by a separate $5 \mathrm{~V}$ power supply. As mentioned, the GSM responds to AT commands which are sent to the module via the microcontroller by programming it using the Arduino IDE. The GSM responds to the AT commands in real time with alittle amount of delay of about 2-3 seconds relying on the command sent. The GSM module has an output buffer. Hence, when data is read from the module the buffer gets cleared, which is why timing each command and reading its response is critical to getting the required response. If both these operations aren't exhausted sync then the required output cannot be procured from the module. The responses from the module are often displayed on the Serial Monitor of the Arduino IDE which forms the thought of interfacing. The AT commands [10] utilized within the proposed device are commands to send and receive texts, obtain the coordinates and make calls. "AT $+\mathrm{CMGF}=1$ " sets the GSM in text mode which is required for both sending and receiving texts. "AT+CMGS=Xxxxxxxxx" sets the mobile number to which text is to be sent. "AT+CNMI $=2,2,0,0,0$ " sets the GSM in text receive mode which responds only a text is received by the module. The coordinates namely 


\section{International Advanced Research Journal in Science, Engineering and Technology}

Vol. 8, Issue 6, June 2021

\section{DOI: $10.17148 / I A R J S E T .2021 .8638$}

latitude and longitude are often obtained using either GPS or GPRS. during this device, GPRS is used since it is more reliable than GPS as GPS requires an open environment. The GPRS content of the module is activated using the command "AT+SAPBR $=1,1$ " and deactivated using "AT+SAPBR $=0,1$ ". Once GPRS is activated the coordinates are obtained using the commands "AT+CIPGSMLOC $=1,1$ ". This also gives this date and time in conjunction with the coordinates. To initiate a call from the module the command "ATDxxxxxxxxxx;" is used. The activation of call mode activates the microphone input and speaker output of the module. When the required devices are connected to those ports within the proper configuration, a user can converse with the recipient.

\section{RESULTS AND DISCUSSION}

This section discusses the results of the three modes of operation of the device and thus the possible inclusions to the device to reinforce its capabilities.

A. Default mode: because the device received a message, a call was initiated to the sender of the message. this happens with a delay of three seconds between the time at which the message was originally sent and thus the instant at which the choice was initiated. the disadvantage of this mode is that if multiple messages are received by the device then only one call is close to move. The person sending the most recent message is patched through with the audio.

B. Location Send mode: As this mode is activated by the push, it takes 7 seconds to urge the coordinates and messages are sent to the trusted contacts within intervals of 4 seconds. The message contains a hyperlink which directs the recipient on to google maps where things of origination of the distress message are getting to be displayed.

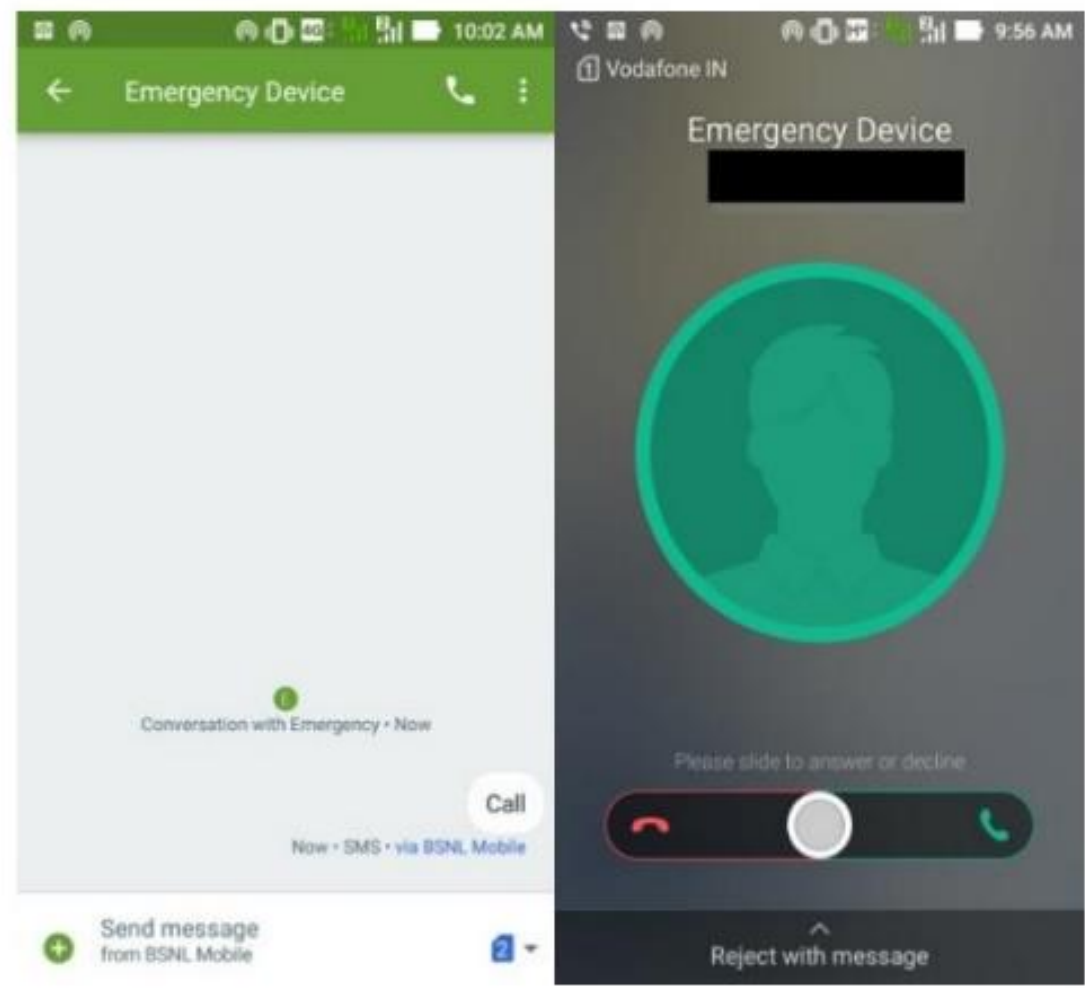

Fig 7. Default mode

C. Calling mode: When this mode is activated by pressing the push, a call to a minimum of one trusted contact is activated within 4 seconds of activation. the disadvantage of this mode is that it's extremely specific and thus the device cannot be programmed to make a call. Thus the success of this mode depends on the availability of the predefined trusted contact. 


\section{International Advanced Research Journal in Science, Engineering and Technology}

Vol. 8, Issue 6, June 2021

\section{DOI: $10.17148 / I A R J S E T .2021 .8638$}

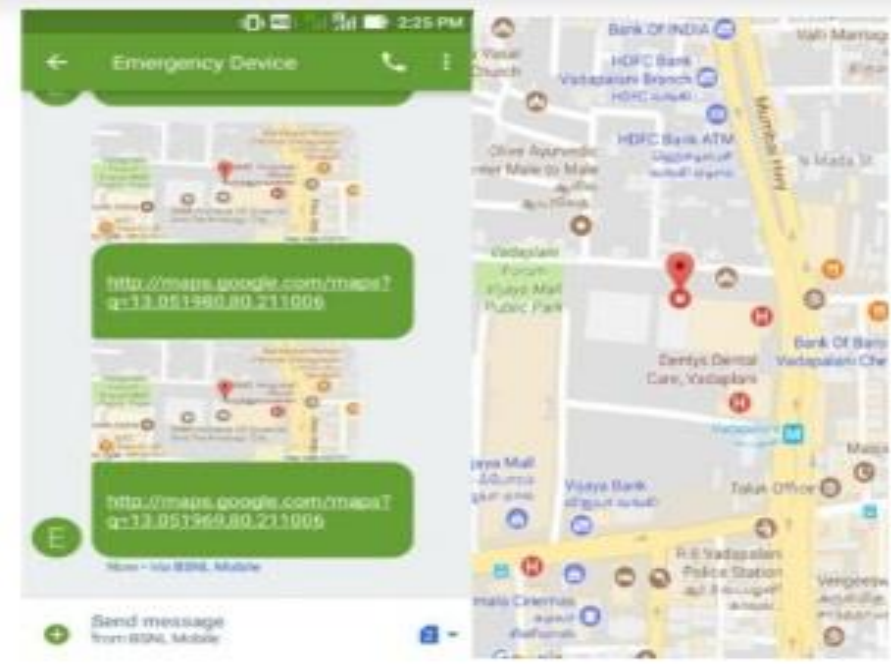

Fig 8. Device sends coordinates to recipient

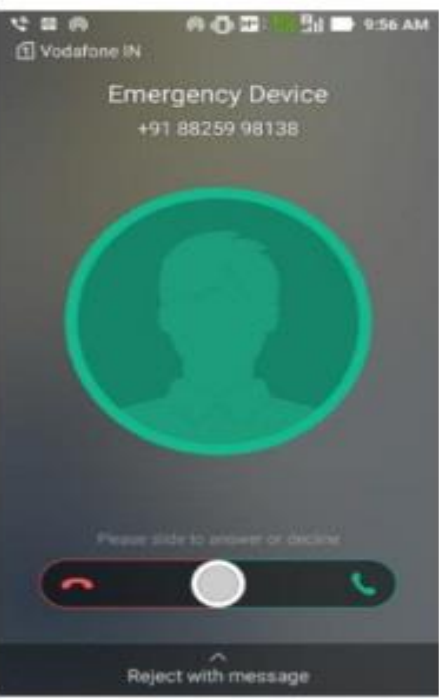

Fig 9. Dialing mode

\section{FUTURE SCOPE}

The addition of a camera module could even be fitted onto the microcontroller and this may be streamed by the GSM module because it's the facility to access the online. The camera can give the detailed surveillance of the environment thus helping in planning out the possible methods of rescue.

\section{CONCLUSION}

The women's guard is that the foremost economical solution for the problems faced by women in India. It provides the trusted contacts with real time location which successively could also be a distress message that makes it possible to prevent major casualties. Replacing the used Arduino Uno with an Arduino Lilypad which can be sewn onto fabrics can help downsize the device. Since it uses low power, rechargeable batteries are often used to make the device more portable.

\section{REFERENCES}

[1]. https://www.poverties.org/blog/violence-againstwomen-in-india.

[2]. https://en.wikipedia.org/wiki/Violence against_women_in_India\#Sexual_crimes.

[3]. Akash Moodbiri, Hamid Shahnasser,"Child Safety Wearable Device”, 2017 International Conference on Information Networking (ICOIN).

[4]. Sangoon Yang, Taehyun Jung," Co-Evolution of Markets for Technology and Markets for Products in Mobile Telecommunication Standards: Examination of Essential Patents for GSM, WCDMA, and LTE Standards", 2014 Proceedings of PICMET '14: Infrastructure and Service Integration.

[5]. Pierre E Hertzog and Arthur J Swart," Arduino - Enabling engineering students to obtain academic success in a design-based module.”,2016 IEEE Global Engineering Education Conference (EDUCON).

[6]. Yusuf Abdullahi Badamasi," The Working Principle Of An Arduino",Eletronics, Computer and Computation(ICECCO), 2014 11th International Conference on Abuja, 2014 pp 1-4.

[7]. https://coreelectronics.com.au/tutorials/arduino-idetutorial.html.

[8]. Bharavi U, Sukesh Rao M," Design and Development of GSM and GPS Tracking Module", 2017 2nd IEEE International Conference On Recent Trends in Electronics Information \& Communication Technology (RTEICT), May 19-20, 2017, India.

[9]. Dmitry Rychkov, Ruy Alberto Pisani Altafim and Reimund Gerhard," Unipolar ferroelectrets - Following the example of the electret microphone more closely", 2014 Annual Report Conference on Electrical Insulation and Dielectric Phenomena.

[10]. https://www.elecrow.com/download/SIM800\%20Serie s_AT\%20Command\%20Manual_V1.09.pdf

[11]. https://www.arduino.cc/en/Tutorial/LiquidCrystalDispl ay.

[12]. Byung Hyung Kim, Sungho Jo, "Deep Physiological Affect Network for the Recognition of Human Emotions", 